DOI 10.4171/JEMS/469

Wojciech Kucharz

\title{
Approximation by continuous rational maps into spheres
}

Received March 19, 2012 and in revised form August 28, 2012

\begin{abstract}
Investigated are continuous rational maps of nonsingular real algebraic varieties into spheres. In some cases, necessary and sufficient conditions are given for a continuous map to be approximable by continuous rational maps. In particular, each continuous map between unit spheres can be approximated by continuous rational maps.
\end{abstract}

Keywords. Real algebraic set, regular map, continuous rational map, semi-algebraic map, approximation

\section{Introduction and main results}

Throughout this section, $X \subseteq \mathbb{R}^{k}$ and $Y \subseteq \mathbb{R}^{l}$ are nonsingular algebraic sets. A map $f: X \rightarrow Y$ is said to be continuous rational if it is continuous and there exist polynomials $P_{1}, \ldots, P_{l}, Q_{1}, \ldots, Q_{l}$ in $\mathbb{R}\left[T_{1}, \ldots, T_{k}\right]$ such that no $Q_{i}$ is identically equal to zero on any irreducible component of $X$ and

$$
f(x)=\left(\frac{P_{1}(x)}{Q_{1}(x)}, \ldots, \frac{P_{l}(x)}{Q_{l}(x)}\right)
$$

for all points $x$ in $X$ with $Q_{1}(x) \neq 0, \ldots, Q_{l}(x) \neq 0$. In other words, besides the continuity of $f$, the existence of a Zariski open and dense subset $U$ of $X$ and a regular map $\varphi: U \rightarrow Y$ with $\left.f\right|_{U}=\varphi$ is required. Since $U$ is dense in $X$ in the Euclidean topology, the map $f$ is completely determined by $\varphi$. In particular, the graph of $f$ is a semi-algebraic set, being the closure of the graph of $\varphi$. Denote by $P(f)$ the indeterminacy locus of the rational map from $X$ into $Y$ represented by $\varphi$. Thus $P(f)$ is the smallest algebraic subset of $X$ for which the restriction map $\left.f\right|_{X \backslash P(f)}: X \backslash P(f) \rightarrow Y$ is regular. It is called the irregularity locus of $f$. Maps with $f(P(f)) \neq Y$ are said to be nice.

Maps with semi-algebraic graphs, called semi-algebraic maps, have been extensively studied in real algebraic geometry (cf. [5]). In problems involving homotopy or approximation, continuous semi-algebraic maps tend to behave like arbitrary continuous maps. On the other hand, regular maps are often too rigid for such problems (cf. [5, 7, 8, 9, 11, $13,14,18,19,20,27])$. Continuous rational maps form a natural intermediate class be-

W. Kucharz: Institute of Mathematics, Faculty of Mathematics and Computer Science, Jagiellonian University, Łojasiewicza 6, 30-348 Kraków, Poland; e-mail: Wojciech.Kucharz@im.uj.edu.pl 
tween regular and semi-algebraic maps, with many specific properties. Probably the first paper in which they are studied in a systematic way is that of the author [26]. It demonstrates that in many interesting cases homotopy classes can be represented by continuous rational maps (cf. [26, Theorems 1.1, 1.2, 1.5, 2.4]). On the other hand, homotopy classes representable by such maps cannot, in general, be represented by regular maps (cf. [5, Sections 13.3, 13.4 and 13.5]). Any continuous rational map which is of class $\mathcal{C}^{\infty}$ is automatically regular [26, Proposition 2.1]. There are continuous rational maps that are not nice [26, Example 2.2]. Contributions of other mathematicians include [17, 24]. In [24], Kollár deals with subtleties involving restrictions and extensions of continuous rational real-valued functions. Interesting algebraic properties of the ring of such functions on $\mathbb{R}^{n}$ are obtained by Fichou, Huisman, Mangolte and Monnier [17].

In the present paper approximation of continuous maps into spheres by continuous rational maps is investigated. The space $\mathcal{C}(X, Y)$ of all continuous maps from $X$ into $Y$ will always be endowed with the compact-open topology. There are the following inclusions:

$$
\mathcal{C}(X, Y) \supseteq \mathcal{R}^{0}(X, Y) \supseteq \mathcal{R}_{0}(X, Y) \supseteq \mathcal{R}(X, Y),
$$

where $\mathcal{R}^{0}(X, Y)$ is the set of all continuous rational maps, $\mathcal{R}_{0}(X, Y)$ consists of the nice maps in $\mathcal{R}^{0}(X, Y)$, and $\mathcal{R}(X, Y)$ is the set of all regular maps. By definition, a continuous map from $X$ into $Y$ can be approximated by continuous rational maps if it belongs to the closure of $\mathcal{R}^{0}(X, Y)$ in $\mathcal{C}(X, Y)$. Approximation by maps of other types is defined in the analogous way.

Assumption. In the rest of this section, the algebraic set $X$ is assumed to be compact.

A continuous map $h: X \rightarrow Y$ is said to be transverse to a point $y_{0}$ in $Y$ if it is smooth (of class $\mathcal{C}^{\infty}$ ) in an open neighborhood $U$ of $h^{-1}\left(y_{0}\right)$ in $X$ and the restriction map $\left.h\right|_{U}: U \rightarrow Y$ is transverse to $y_{0}$ in the usual sense. In that case, $h^{-1}\left(y_{0}\right)$ is a smooth submanifold of $X$. If $h$ is transverse to $y_{0}$ and $h^{-1}\left(y_{0}\right)=\operatorname{Reg}(V)$ for some algebraic subset $V$ of $X$, then $h$ is said to be adapted to $y_{0}$. Furthermore, $h$ is said to be adapted if it is adapted to some point $y$ in $Y$. Here $\operatorname{Reg}(V)$ stands for the set of nonsingular points of $V$. In particular, $\operatorname{dim} h^{-1}\left(y_{0}\right)=\operatorname{dim} V$ and $V \backslash \operatorname{Reg}(V)$ is an algebraic subset of $X$, which is either empty or satisfies $\operatorname{dim}(V \backslash \operatorname{Reg}(V))<\operatorname{dim} V$. If the set $h^{-1}(y)$ is empty, then $h$ is adapted to $y$.

The notions just introduced are natural when nice continuous rational maps are considered.

Proposition 1.1. Any nice continuous rational map from $X$ into $Y$ is adapted.

Proof. Let $f: X \rightarrow Y$ be a nice continuous rational map. Since $f(P(f))$ is a proper compact subset of $Y$, it follows from Sard's theorem that the regular map $\left.f\right|_{X \backslash P(f)}$ : $X \backslash P(f) \rightarrow Y$ is transverse to some point $y_{0}$ in $Y \backslash f(P(f))$. Hence $f^{-1}\left(y_{0}\right)$ is a nonsingular Zariski closed subset of $X \backslash P(f)$. If $V$ is the Zariski closure of $f^{-1}\left(y_{0}\right)$ in $X$, then $V \backslash f^{-1}\left(y_{0}\right)$ is a Zariski closed subset of $X$ contained in $P(f)$, with $\operatorname{dim}\left(V \backslash f^{-1}\left(y_{0}\right)\right)<\operatorname{dim} f^{-1}\left(y_{0}\right)$. Hence $f^{-1}\left(y_{0}\right)=\operatorname{Reg}(V)$, the set $f^{-1}\left(y_{0}\right)$ being compact. Consequently, $f$ is adapted to $y_{0}$. 
It is convenient to relax the notion of "adapted" in a suitable way. This requires some preparation. For any smooth manifolds $N$ and $P$, let $\mathcal{C}^{\infty}(N, P)$ denote the space of all smooth maps from $N$ into $P$ endowed with the $\mathcal{C}^{\infty}$ topology (cf. [22]). The source manifold will always be assumed to be compact, and hence the weak $\mathcal{C}^{\infty}$ topology coincides with the strong one.

A compact smooth submanifold $M$ of $X$ is said to admit a weak algebraic approximation in $X$ if each neighborhood of the inclusion map $M \hookrightarrow X$ in the space $\mathcal{C}^{\infty}(M, X)$ contains a smooth embedding $e: M \rightarrow X$ with $e(M)=\operatorname{Reg}(V)$ for some algebraic subset $V$ of $X$. A continuous map $h: X \rightarrow Y$ is said to be weakly adapted to a point $y_{0}$ in $Y$ if it is transverse to $y_{0}$ and the smooth submanifold $h^{-1}\left(y_{0}\right)$ of $X$ admits a weak algebraic approximation in $X$. Furthermore, $h$ is said to be weakly adapted if it is weakly adapted to some point $y$ in $Y$.

In what follows, mostly maps with values in the unit $p$-sphere

$$
\mathbb{S}^{p}:=\left\{\left(u_{1}, \ldots, u_{p+1}\right) \in \mathbb{R}^{p+1} \mid u_{1}^{2}+\cdots+u_{p+1}^{2}=1\right\}
$$

are considered. The main result, whose proof is postponed until Section 2, is Theorem 1.2. Its significance is elaborated upon in the remainder of this section.

Theorem 1.2. For a continuous map $h: X \rightarrow \mathbb{S}^{p}$, the following conditions are equivalent:

(a) $h$ can be approximated by nice continuous rational maps with irregularity locus of dimension at most $\operatorname{dim} X-p$.

(b) $h$ can be approximated by nice continuous rational maps.

(c) h can be approximated by adapted continuous maps.

(d) $h$ can be approximated by adapted smooth maps.

(e) $h$ can be approximated by weakly adapted continuous maps.

(f) $h$ can be approximated by weakly adapted smooth maps.

Theorem 1.2 immediately implies the following:

Corollary 1.3. If $\operatorname{dim} X=p$, then the set $\mathcal{R}_{0}\left(X, \mathbb{S}^{p}\right)$ is dense in $\mathcal{C}\left(X, \mathbb{S}^{p}\right)$.

Proof. According to Sard's theorem, each smooth map $g: X \rightarrow \mathbb{S}^{p}$ is transverse to some point $z_{0}$ in $\mathbb{S}^{p}$. Since in the case under consideration the set $g^{-1}\left(z_{0}\right)$ is finite, the map $g$ is adapted to $z_{0}$. It suffices to apply Theorem 1.2.

In Corollary 1.3 , the set $\mathcal{R}_{0}\left(X, \mathbb{S}^{p}\right)$ cannot be replaced by $\mathcal{R}\left(X, \mathbb{S}^{p}\right)$. Indeed, a continuous map from $\mathbb{S}^{1} \times \mathbb{S}^{1}$ into $\mathbb{S}^{2}$ can be approximated by regular maps if and only if it is null homotopic (cf. [11, Proposition 2.2, Example 2.1]).

Corollary 1.4. If each compact smooth submanifold of $X$, of codimension $p$ and with trivial normal bundle, admits a weak algebraic approximation in $X$, then the set $\mathcal{R}_{0}\left(X, \mathbb{S}^{p}\right)$ is dense in $\mathcal{C}\left(X, \mathbb{S}^{p}\right)$.

Proof. By Sard's theorem, each smooth map $g: X \rightarrow \mathbb{S}^{p}$ is transverse to some point $z_{0}$ in $\mathbb{S}^{p}$. Hence the set $g^{-1}\left(z_{0}\right)$ is either empty or it is a smooth submanifold of $X$ of codimension $p$ and with trivial normal bundle. Consequently, $g$ is weakly adapted to $z_{0}$. The proof is complete in view of Theorem 1.2.

Corollary 1.4 is crucial in the proof of the following result. 
Theorem 1.5. For any pair $(n, p)$ of nonnegative integers, the set $\mathcal{R}_{0}\left(\mathbb{S}^{n}, \mathbb{S}^{p}\right)$ is dense in $\mathcal{C}\left(\mathbb{S}^{n}, \mathbb{S}^{p}\right)$.

Proof. Let $M$ be a compact smooth submanifold of $\mathbb{S}^{n}$ with $\operatorname{dim} M<n$. Let $a$ be a point in $\mathbb{S}^{n} \backslash M$ and let $\rho: \mathbb{S}^{n} \backslash\{a\} \rightarrow \mathbb{R}^{n}$ be the stereographic projection. Clearly, $\rho$ is a biregular isomorphism. According to [1, Theorem A] (cf. also [2, Theorem 2.10.5 and Corollary 2.10.6]), the smooth submanifold $\rho(M)$ of $\mathbb{R}^{n}$ admits a weak algebraic approximation in $\mathbb{R}^{n}$, and hence $M$ admits a weak algebraic approximation in $\mathbb{S}^{n}$. In view of Corollary 1.4 , the proof is complete for $p \geq 1$. The case $p=0$ is obvious.

A stronger result holds for pairs $(n, p)$ with $p$ equal to 1,2 or 4 , namely, the set $\mathcal{R}\left(\mathbb{S}^{n}, \mathbb{S}^{p}\right)$ is dense in $\mathcal{C}\left(\mathbb{S}^{n}, \mathbb{S}^{p}\right)$ (cf. [6] or [5, Theorem 13.3.10]). If $n<p$, then $\mathcal{R}\left(\mathbb{S}^{n}, \mathbb{S}^{p}\right)$ is dense in $\mathcal{C}\left(\mathbb{S}^{n}, \mathbb{S}^{p}\right)$ for simple reasons (cf. Lemma 2.3). No pair $(n, p)$ is known with $\mathcal{R}\left(\mathbb{S}^{n}, \mathbb{S}^{p}\right)$ dense in $\mathcal{C}\left(\mathbb{S}^{n}, \mathbb{S}^{p}\right), n \geq p$ and $p$ different from $0,1,2$ and 4 . According to [26, Theorem 1.1], for any pair $(n, p)$, each continuous map from $\mathbb{S}^{n}$ into $\mathbb{S}^{p}$ is homotopic to a continuous rational map $f: \mathbb{S}^{n} \rightarrow \mathbb{S}^{p}$ with $f(P(f))$ containing at most one point. Moreover, for any nonnegative integer $r$, such a map $f$ can be chosen to be of class $\mathcal{C}^{r}$.

In general $\mathcal{R}^{0}\left(X, \mathbb{S}^{p}\right)$ need not be dense in $\mathcal{C}\left(X, \mathbb{S}^{p}\right)$. Simple obstructions can be expressed in terms of homology or cohomology classes representable by algebraic subsets. This is made precise below.

A cohomology class in $H^{c}(X ; \mathbb{Z} / 2)$ is said to be algebraic if it corresponds via the Poincaré duality isomorphism to a homology class in $H_{d}(X ; \mathbb{Z} / 2)$ represented by a $d$ dimensional algebraic subset of $X$ with $d=\operatorname{dim} X-c$ (cf. $[3,5,12,15])$. The set $H_{\text {alg }}^{c}(X ; \mathbb{Z} / 2)$ of all algebraic cohomology classes in $H^{c}(X ; \mathbb{Z} / 2)$ forms a subgroup. The groups $H_{\text {alg }}^{c}(-; \mathbb{Z} / 2)$ have the following functorial property: If also $Y$ is compact and $f: X \rightarrow Y$ is a continuous rational map, then

$$
f^{*}\left(H_{\text {alg }}^{c}(Y ; \mathbb{Z} / 2)\right) \subseteq H_{\text {alg }}^{c}(X ; \mathbb{Z} / 2),
$$

where $f^{*}: H^{c}(Y ; \mathbb{Z} / 2) \rightarrow H^{c}(X ; \mathbb{Z} / 2)$ is the homomorphism induced by $f$ (cf. [26, Proposition 1.3]). If a continuous map $h: X \rightarrow Y$ can be approximated by continuous rational maps, then it is homotopic to a continuous rational map, and hence the last inclusion holds with $f$ replaced by $h$. In some cases, the converse is true.

Let $s_{p}$ denote the unique generator of the group $H_{\text {alg }}^{p}\left(\mathbb{S}^{p} ; \mathbb{Z} / 2\right)=H^{p}\left(\mathbb{S}^{p} ; \mathbb{Z} / 2\right) \cong$ $\mathbb{Z} / 2$ if $p \geq 1$, and one of the two generators if $p=0$.

Example 1.6. For a continuous map $h: X \rightarrow \mathbb{S}^{1}$, the following conditions are equivalent:

(a) $h$ is homotopic to a continuous rational map.

(b) $h$ can be approximated by regular maps.

(c) $h^{*}\left(s_{1}\right)$ is in $H_{\text {alg }}^{1}(X ; \mathbb{Z} / 2)$.

The equivalence of (b) and (c) is proved in [6] (cf. also [5, Theorems 12.4.6, 13.3.1]). As explained above, (a) implies (c). Clearly, (a) follows from (b). It is well known that (c) imposes essential restrictions on $h$ (cf. [9] or [5, pp. 354-356]). 
Theorem 1.7. If $\operatorname{dim} X=p+1$, then for a continuous map $h: X \rightarrow \mathbb{S}^{p}$, the following conditions are equivalent:

(a) $h$ can be approximated by nice continuous rational maps.

(b) $h$ is homotopic to a continuous rational map.

(c) $h^{*}\left(s_{p}\right)$ is in $H_{\text {alg }}^{p}(X ; \mathbb{Z} / 2)$.

Proof. The case $p=0$ is obvious. Indeed, in this case, each of the conditions (a), (b) and (c) implies that $h$ is constant on each irreducible component of $X$, and hence $h$ is a regular map. For $p=1$, Theorem 1.7 is a special case of Example 1.6. Therefore in what follows $p \geq 2$.

Clearly, (a) implies (b), and (b) implies (c). Suppose that (c) is satisfied. In the proof that (a) holds, the map $h$ can be assumed to be smooth. Let $B$ be an algebraic (possibly singular) curve in $X$ whose homology class in $H_{1}(X ; \mathbb{Z} / 2)$ is Poincare dual to the cohomology class $h^{*}\left(s_{p}\right)$. By Sard's theorem, $h$ is transverse to some point $z_{0}$ in $\mathbb{S}^{p} \backslash h(B)$. The homology class in $H_{1}(X ; \mathbb{Z} / 2)$ represented by the smooth curve $h^{-1}\left(z_{0}\right)$ is Poincare dual to $h^{*}\left(s_{p}\right)$ (cf. [15, Proposition 2.15]). Consequently, $B$ and $h^{-1}\left(z_{0}\right)$ represent the same homology class. This property implies that the smooth curve $h^{-1}\left(z_{0}\right)$ admits a weak algebraic approximation in $X$ (cf. [26, proof of (c) $\Rightarrow$ (a) in Theorem 1.5]), and hence the map $h$ is weakly adapted to $z_{0}$. Condition (a) is satisfied in view of Theorem 1.2.

It is worthwhile to illustrate Theorem 1.7 with an example. Let $\mathbb{T}^{n}=\mathbb{S}^{1} \times \cdots \times \mathbb{S}^{1}$ be the $n$-fold product of $\mathbb{S}^{1}$.

Example 1.8. Since $H_{\mathrm{alg}}^{l}\left(\mathbb{T}^{n} ; \mathbb{Z} / 2\right)=H^{l}\left(\mathbb{T}^{n} ; \mathbb{Z} / 2\right)$ for all $l \geq 0$, according to Theorem 1.7, the set $\mathcal{R}_{0}\left(\mathbb{T}^{p+1}, \mathbb{S}^{p}\right)$ is dense in $\mathcal{C}\left(\mathbb{T}^{p+1}, \mathbb{S}^{p}\right)$. On the other hand, if $n>p \geq 1$ and $h: \mathbb{T}^{n} \rightarrow \mathbb{S}^{p}$ is a continuous map with $h^{*}\left(s_{p}\right) \neq 0$ in $H^{p}\left(\mathbb{T}^{n} ; \mathbb{Z} / 2\right)$, then there exist a nonsingular real algebraic set $Z$ and a smooth diffeomorphism $\varphi: Z \rightarrow \mathbb{T}^{n}$ such that the map $h \circ \varphi: Z \rightarrow \mathbb{S}^{p}$ is not homotopic to a continuous rational map (cf. Theorem 2.8 in Section 2). Furthermore, if $p$ is even, then $h$ is not homotopic to a regular map, and hence it cannot be approximated by regular maps. This last assertion can be justified as follows. In [4], for any nonnegative integer $k$ and any real algebraic set $V$, a subgroup $H_{\mathbb{C} \text {-alg }}^{2 k}(V ; \mathbb{Z})$ of the cohomology group $H^{2 k}(V ; \mathbb{Z})$ is defined. If $f: V \rightarrow W$ is a regular map between real algebraic sets, then

$$
f^{*}\left(H_{\mathbb{C} \text {-alg }}^{2 k}(W ; \mathbb{Z})\right) \subseteq H_{\mathbb{C} \text {-alg }}^{2 k}(V ; \mathbb{Z}) .
$$

According to [4, Proposition 4.8], $H_{\mathbb{C} \text {-alg }}^{2 k}\left(\mathbb{S}^{2 k} ; \mathbb{Z}\right)=H^{2 k}\left(\mathbb{S}^{2 k} ; \mathbb{Z}\right)$. It follows directly from the definition that $H_{\mathbb{C} \text {-alg }}^{2 k}\left(\mathbb{T}^{n} ; \mathbb{Z}\right)=0$ for all $k \geq 1$. Consequently, for any regular map $g: \mathbb{T}^{n} \rightarrow \mathbb{S}^{2 k}$,

$$
g^{*}\left(H^{2 k}\left(\mathbb{S}^{2 k} ; \mathbb{Z}\right)\right)=0 \quad \text { in } H^{2 k}\left(\mathbb{T}^{n} ; \mathbb{Z}\right)
$$

for all $k \geq 1$. If $h: \mathbb{T}^{n} \rightarrow \mathbb{S}^{2 k}$ is a continuous map with $h^{*}\left(s_{2 k}\right) \neq 0$ in $H^{2 k}\left(\mathbb{T}^{n} ; \mathbb{Z} / 2\right)$, then $\left.h^{*}\left(H^{2 k}\left(\mathbb{S}^{2 k} ; \mathbb{Z}\right)\right)\right) \neq 0$ in $H^{2 k}\left(\mathbb{T}^{n} ; \mathbb{Z}\right)$, and hence $h$ cannot be homotopic to a regular map. The assertion is proved.

It remains an open problem whether or not the sets $\mathcal{R}_{0}\left(\mathbb{T}^{n}, \mathbb{S}^{p}\right)$ and $\mathcal{R}^{0}\left(\mathbb{T}^{n}, \mathbb{S}^{p}\right)$ are dense in $\mathcal{C}\left(\mathbb{T}^{n}, \mathbb{S}^{p}\right)$ for all $n$ and $p$. 


\section{Proofs and further results}

The language of real algebraic geometry, as in [5], is used throughout this section. The term real algebraic variety designates a locally ringed space isomorphic to an algebraic subset of $\mathbb{R}^{n}$, for some $n$, endowed with the Zariski topology and the sheaf of real-valued regular functions (such objects are called affine real algebraic varieties in [5]). The quasiprojective real algebraic varieties are real algebraic varieties in this sense (cf. [5, Proposition 3.2.10, Theorem 3.4.4]). Zariski closed subsets of a real algebraic variety are often called algebraic subsets. Morphisms between real algebraic varieties are called regular maps. Every real algebraic variety carries also the Euclidean topology, which is determined by the usual metric on $\mathbb{R}$. Unless explicitly stated otherwise, all topological notions relating to real algebraic varieties refer to the Euclidean topology.

For any real algebraic variety $X$, algebraic vector subbundles of the trivial $\mathbb{R}$-vector bundle $\varepsilon_{X}^{k}$ with total space $X \times \mathbb{R}^{k}$, for some $k$, are called algebraic vector bundles on $X$. In other words, algebraic vector bundles on $X$ correspond to finitely generated projective modules over the ring of regular functions from $X$ into $\mathbb{R}$ (cf. [5] for other equivalent definitions).

Lemma 2.1. Let $X$ be a compact real algebraic variety and let $C$ be an algebraic subset of $X$. Let $\xi$ be an algebraic vector bundle on $X$ and let $\sigma: X \rightarrow \xi$ be a continuous section of $\xi$, whose restriction $\left.\sigma\right|_{C}$ is an algebraic section. Let $\mathcal{U}$ be a neighborhood of $\sigma$ in the space of all continuous sections of $\xi$, endowed with the compact-open topology. Then there exists an algebraic section $s: X \rightarrow \xi$ that belongs to $\mathcal{U}$ and satisfies $\left.s\right|_{C}=\left.\sigma\right|_{C}$.

Proof. This is a special case of [10, Lemma 2.2]. It should be mentioned that algebraic vector bundles in the sense of this paper are called strongly algebraic in [10].

For any algebraic vector subbundle $\eta$ of $\varepsilon_{X}^{k}$, let $\eta^{\perp}$ denote its orthogonal complement in $\varepsilon_{X}^{k}$, with respect to the standard scalar product on $\mathbb{R}^{k}$. Then $\eta^{\perp}$ is an algebraic subbundle of $\varepsilon_{X}^{k}$ and

$$
\eta \oplus \eta^{\perp}=\varepsilon_{X}^{k} .
$$

Suppose that $X$ is compact and nonsingular. A topological line bundle $\lambda$ on $X$ is topologically isomorphic to an algebraic line bundle if and only if its first Stiefel-Whitney class $w_{1}(\lambda)$ is in $H_{\text {alg }}^{1}(X ; \mathbb{Z} / 2$ ) (cf. [5, Theorem 12.4.6]).

The following approximation result for maps with values in real projective $n$-space $\mathbb{P}^{n}(\mathbb{R})$ will be very useful (it is a relative version of [5, Theorem 13.3.1] with $m=n+1$, $p=1$ and $\mathbb{F}=\mathbb{R})$.

Lemma 2.2. Let $X$ be a compact nonsingular real algebraic variety and let $A$ be an algebraic subset of $X$. Let $f: X \rightarrow \mathbb{P}^{n}(\mathbb{R})$ be a continuous map whose restriction $\left.f\right|_{A}: A \rightarrow \mathbb{P}^{n}(\mathbb{R})$ is a regular map. Assume that

$$
f^{*}\left(H^{1}\left(\mathbb{P}^{n}(\mathbb{R}) ; \mathbb{Z} / 2\right)\right) \subseteq H_{\text {alg }}^{1}(X ; \mathbb{Z} / 2) .
$$

Then each neighborhood of $f$ in $\mathcal{C}\left(X, \mathbb{P}^{n}(\mathbb{R})\right)$ contains a regular map $g: X \rightarrow \mathbb{P}^{n}(\mathbb{R})$ with $\left.g\right|_{A}=\left.f\right|_{A}$. 
Proof. Let $\gamma_{n}$ denote the universal line bundle on $\mathbb{P}^{n}(\mathbb{R})$. Recall that

$$
\left\{(l, e) \in \mathbb{P}^{n}(\mathbb{R}) \times \mathbb{R}^{n+1} \mid e \in l\right\}
$$

is the total space of $\gamma_{n}$. Here $\mathbb{P}^{n}(\mathbb{R})$ is regarded as the space of 1-dimensional vector subspaces of $\mathbb{R}^{n+1}$. In particular, $\gamma_{n}$ is an algebraic vector subbundle of $\varepsilon_{\mathbb{P}^{n}(\mathbb{R})}^{n+1}$. By assumption, the cohomology class $w_{1}\left(f^{*} \gamma_{n}\right)=f^{*}\left(w_{1}\left(\gamma_{n}\right)\right)$ is in $H_{\text {alg }}^{1}(X ; \mathbb{Z} / 2)$, and hence there exist an algebraic line bundle $\lambda$ on $X$ and a topological isomorphism $\varphi: \lambda \rightarrow f^{*} \gamma_{n}$. Let $\sigma: X \rightarrow \operatorname{Hom}\left(\lambda, f^{*} \gamma_{n}\right)$ be the continuous section defined by $\sigma(x)(w)=\varphi(w)$ for all $x$ in $X$ and all $w$ in the fiber $\lambda_{x}$ of $\lambda$ over $x$. Regarding $f^{*} \gamma_{n}$ and $f^{*} \gamma_{n}^{\perp}$ as topological subbundles of $\varepsilon_{X}^{n+1}$, one notes that the map

$$
\psi: f^{*} \gamma_{n} \oplus f^{*} \gamma_{n}^{\perp} \rightarrow \varepsilon_{X}^{n+1}
$$

defined by $\psi(u, v)=u+v$ for all $u$ in $\left(f^{*} \gamma_{n}\right)_{x}$ and $v$ in $\left(f^{*} \gamma_{n}^{\perp}\right)_{x}$ with $x$ in $X$ is an isomorphism of topological vector bundles, which becomes a biregular isomorphism of algebraic vector bundles when the bundles are restricted to $A$ (here the regularity of $\left.f\right|_{A}$ is used). Hence the map $\pi: \varepsilon_{X}^{n+1} \rightarrow f^{*} \gamma_{n}$ determined by $\pi(\psi(u, v))=u$ is a continuous homomorphism of vector bundles, which becomes a regular homomorphism when the bundles are restricted to $A$. Consider the continuous section $\tau: X \rightarrow$ $\operatorname{Hom}\left(\lambda, \varepsilon_{X}^{n+1}\right)$ defined by $\tau(x)(w)=\psi(\sigma(x)(w), 0)$. Since $\operatorname{Hom}\left(\lambda, \varepsilon_{X}^{n+1}\right)$ is an algebraic vector bundle, according to Lemma 2.1 (with $C=\emptyset$ ), there exists an algebraic section $t: X \rightarrow \operatorname{Hom}\left(\lambda, \varepsilon_{X}^{n+1}\right)$ arbitrarily close to $\tau$. Then the continuous section $\sigma_{1}: X \rightarrow \operatorname{Hom}\left(\lambda, f^{*} \gamma_{n}\right)$ defined by $\sigma_{1}(x)(w)=\pi(t(x)(w))$ is close to $\sigma$, and $\left.\sigma_{1}\right|_{A}$ is an algebraic section of

$$
\left.\operatorname{Hom}\left(\lambda, f^{*} \gamma_{n}\right)\right|_{A}=\operatorname{Hom}\left(\left.\lambda\right|_{A},\left(\left.f\right|_{A}\right)^{*} \gamma_{n}\right)
$$

Define a continuous section $\tau_{1}: X \rightarrow \operatorname{Hom}\left(\lambda, \varepsilon_{X}^{n+1}\right)$ by $\tau_{1}(x)(w)=\psi\left(\sigma_{1}(x)(w), 0\right)$. By construction, $\tau_{1}$ is close to $\tau$, and $\left.\tau_{1}\right|_{A}$ is an algebraic section of $\left.\operatorname{Hom}\left(\lambda, \varepsilon_{X}^{n+1}\right)\right|_{A}$. According to Lemma 2.1 (with $C=A$ ), there exists an algebraic section $s: X \rightarrow \operatorname{Hom}\left(\lambda, \varepsilon_{X}^{n+1}\right.$ ) close to $\tau_{1}$ and with $\left.s\right|_{A}=\left.\tau_{1}\right|_{A}$. Since $X$ is compact, one may assume that the linear transformation $s(x): \lambda_{x} \rightarrow\left(\varepsilon_{X}^{n+1}\right)_{x}$ is injective for all points $x$ in $X$. Define $g: X \rightarrow \mathbb{P}^{n}(\mathbb{R})$ by $g(x)=\rho\left(s(x)\left(\lambda_{x}\right)\right)$ for $x$ in $X$, where $\rho: X \times \mathbb{R}^{n+1} \rightarrow \mathbb{R}^{n+1}$ is the canonical projection. By [5, Proposition 3.4.7], $g$ is a regular map. Moreover, $\left.g\right|_{A}=\left.f\right|_{A}$ and $g$ is close to $f$.

It is convenient to state explicitly the following simple fact.

Lemma 2.3. Let $Z$ be a compact real algebraic variety (not necessarily nonsingular) and let $h: Z \rightarrow \mathbb{S}^{p}$ be a continuous map. If either $h$ is not surjective or $\operatorname{dim} Z<p$, then $h$ can be approximated by regular maps.

Proof. Suppose that $h$ is not surjective and let $a$ be a point in $\mathbb{S}^{p} \backslash h(Z)$. The stereographic projection $\rho: \mathbb{S}^{p} \backslash\{a\} \rightarrow \mathbb{R}^{p}$ is a biregular isomorphism, and hence $h$ can be approximated by regular maps in view of the Weierstrass approximation theorem. 
The case $\operatorname{dim} Z<p$ can readily be reduced to the previous one. First choose a continuous semi-algebraic map $g: Z \rightarrow \mathbb{S}^{p}$ close to $h$. It suffices to note that $\operatorname{dim} g(Z) \leq \operatorname{dim} Z$ (cf. [5, Theorem 2.8.8]), and hence $g$ is not surjective.

Let $N$ and $P$ be smooth manifolds, and let $A$ be a closed subset of $N$. A map $f: A \rightarrow P$ is said to be smooth if it has a smooth extension to an open neighborhood of $A$ in $N$.

The next technical result is stated in the form sufficient for the purposes of this paper. It could easily be generalized to maps between arbitrary smooth manifolds.

Lemma 2.4. Let $X$ be a compact nonsingular real algebraic variety and let $S$ be a closed subset of $X$. Let $h: X \rightarrow \mathbb{S}^{p}$ be a continuous map whose restriction $\left.h\right|_{S}: S \rightarrow \mathbb{S}^{p}$ is a smooth map. Let $\mathcal{U}$ be an open neighborhood of h in $\mathcal{C}\left(X, \mathbb{S}^{p}\right)$. Assume that $h$ is transverse to a point $z_{0}$ in $\mathbb{S}^{p}$ with $h^{-1}\left(z_{0}\right) \cap S=\emptyset$. Then there exists a neighborhood $\mathcal{V}$ of $\left.h\right|_{S}$ in $\mathcal{C}\left(S, \mathbb{S}^{p}\right)$ such that, for each smooth map $r: S \rightarrow \mathbb{S}^{p}$ in $\mathcal{V}$, one can find a smooth map $h_{r}: X \rightarrow \mathbb{S}^{p}$ in $\mathcal{U}$ with $h_{r}^{-1}\left(z_{0}\right)=h^{-1}\left(z_{0}\right), h_{r}=h$ in a neighborhood of $h^{-1}\left(z_{0}\right)$ in $X$ and $\left.h_{r}\right|_{S}=r$.

Proof. The set $\mathbb{R}^{p+1} \backslash\{0\}$ together with radial projection onto $\mathbb{S}^{p}$ can be regarded as a tubular neighborhood of $\mathbb{S}^{p}$ in $\mathbb{R}^{p+1}$. This fact and the existence of smooth partitions of unity imply that all the constructions described below are possible.

Let $K$ be a compact neighborhood of $S$ in $X$ with $h(K) \subseteq \mathbb{S}^{p} \backslash\left\{z_{0}\right\}$. Choose an open neighborhood $U$ of $h^{-1}\left(z_{0}\right)$ in $X \backslash K$ in which the map $h$ is smooth. Since $\left.h\right|_{S}$ has a smooth extension to an open neighborhood of $S$ in $X$, by slightly modifying $h$ on $K \backslash S$, one obtains a continuous map $g_{1}: X \rightarrow \mathbb{S}^{p}$ in $\mathcal{U}$ such that $g_{1}=h$ on $(X \backslash K) \cup S$, $g_{1}(K) \subseteq \mathbb{S}^{p} \backslash\left\{z_{0}\right\}$ and $g_{1}$ is smooth in an open neighborhood of $S$ in $X$. Note that $g_{1}^{-1}\left(z_{0}\right)=h^{-1}\left(z_{0}\right)$. Since $U$ is contained in $X \backslash K$, the map $g_{1}$ is smooth in $U$. In view of the compactness of $X$, there exists a compact neighborhood $L$ of $z_{0}$ in $\mathbb{S}^{p}$ with $g_{1}^{-1}(L) \subseteq U$. Let $U_{1}$ be an open neighborhood of $g_{1}^{-1}\left(z_{0}\right)$ in $X$, contained in the interior of $g_{1}^{-1}(L)$. By slightly modifying $g_{1}$ on $X \backslash\left(g_{1}^{-1}(L) \cup S\right)$, one obtains a smooth map $g_{2}$ in $\mathcal{U}$ such that $g_{2}=g_{1}$ on $g_{1}^{-1}(L) \cup S$ and $g_{2}\left(X \backslash U_{1}\right) \subseteq \mathbb{S}^{p} \backslash\left\{z_{0}\right\}$. Note that $g_{2}^{-1}\left(z_{0}\right)=$ $g_{1}^{-1}\left(z_{0}\right)=h^{-1}\left(z_{0}\right)$. Replacing $h$ by $g_{2}$, one may assume without loss of generality that the map $h$ is smooth.

If $\mathcal{V}$ is a sufficiently small neighborhood of $\left.h\right|_{S}$ in $\mathcal{C}\left(S, \mathbb{S}^{p}\right)$, then given a smooth map $r: S \rightarrow \mathbb{S}^{p}$ in $\mathcal{V}$, one can slightly modify $h$ on $K$ and construct a smooth map $h_{r}: X \rightarrow \mathbb{S}^{p}$ in $\mathcal{U}$ with $h_{r}=h$ on $X \backslash K$ and $h_{r}(K) \subseteq \mathbb{S}^{p} \backslash\left\{z_{0}\right\}$. Such a smooth map $h_{r}$ satisfies all the requirements.

In the proof of the main result of this section, Theorem 2.5 below, blowups play a prominent role. Let $X$ be a nonsingular real algebraic variety and let $Z$ be a nonsingular algebraic subset of $X$. The blowup of $X$ at $Z$ will be denoted by $\pi(X, Z): B(X, Z) \rightarrow X$. Recall that $\pi(X, Z)$ induces a biregular isomorphism from $B(X, Z) \backslash \pi(X, Z)^{-1}(Z)$ onto $X \backslash Z$. Moreover, $\pi(X, Z)^{-1}(Z)$ is the projectivization of the normal bundle to $Z$ in $X$.

Let $S$ be an algebraic (possibly singular) subset of $X$. Suppose there is a finite sequence of maps

$$
Y=X_{k} \stackrel{\pi_{k}}{\rightarrow} X_{k-1} \stackrel{\pi_{k-1}}{\longrightarrow} \cdots \stackrel{\pi_{2}}{\longrightarrow} X_{1} \stackrel{\pi_{1}}{\rightarrow} X_{0}=X
$$


where $\pi_{1}$ is the blowup of $X_{0}$ at a nonsingular algebraic subset of $X_{0}$ contained in $S$ and $\pi_{i+1}$ is the blowup of $X_{i}$ at a nonsingular algebraic subset of $X_{i}$ contained in $\left(\pi_{1} \circ \cdots \circ \pi_{i}\right)^{-1}(S)$ for $i=1, \ldots, k-1$. The composite map $\pi=\pi_{1} \circ \cdots \circ \pi_{k}: Y \rightarrow X$ will be called a multiblowup of $X$ over $S$. Note that $Y$ is a nonsingular real algebraic variety and the restriction $\pi_{S}: Y \backslash \pi^{-1}(S) \rightarrow X \backslash S$ of $\pi$ is a biregular isomorphism. Furthermore, $\pi$ is a proper map.

Theorem 2.5. Let $X$ be a compact nonsingular real algebraic variety and let $h: X \rightarrow \mathbb{S}^{p}$ be a continuous map. Assume that $h$ is adapted to a point $z_{0}$ in $\mathbb{S}^{p}$ and $h^{-1}\left(z_{0}\right)=\operatorname{Reg}(V)$ for some algebraic subset $V$ of $X$. If the restriction of $h$ to the algebraic subset $S:=$ $V \backslash \operatorname{Reg}(V)$ of $X$ is a regular map, then each open neighborhood $\mathcal{U}$ of $h$ in $\mathcal{C}\left(X, \mathbb{S}^{p}\right)$ contains a nice continuous rational map $f: X \rightarrow \mathbb{S}^{p}$ with $P(f) \subseteq V$ and $f(V)=h(V)$.

Proof. Any regular map from $S$ into $\mathbb{S}^{p}$ has a smooth extension to some open neighborhood of $S$ in $X$. Hence in view of Lemma 2.4, the map $h$ can be assumed to be smooth.

By Hironaka's resolution of singularities theorem [21] (cf. also [23] for a very readable exposition), there exists a multiblowup $\pi: Y \rightarrow X$ of $X$ over $S$ such that the Zariski closure $W$ of $\pi^{-1}(\operatorname{Reg}(V))$ in $Y$ is nonsingular. Note that

$$
W=\pi^{-1}(\operatorname{Reg}(V))=\pi^{-1}\left(h^{-1}\left(z_{0}\right)\right) .
$$

Indeed, since $W$ is nonsingular, the set $\pi^{-1}(\operatorname{Reg}(V))$ is dense in $W$ in the Euclidean topology. Hence the closedness of $\operatorname{Reg}(V)$ in $X$ implies the inclusion $\pi(W) \subseteq \operatorname{Reg}(V)$, from which (1) follows. In particular,

$$
W \cap \pi^{-1}(S)=\emptyset .
$$

Since the restriction map $\pi_{S}: Y \backslash \pi^{-1}(S) \rightarrow X \backslash S$ of $\pi$ is a biregular isomorphism, the smooth map $h \circ \pi: Y \rightarrow \mathbb{S}^{p}$ is transverse to $z_{0}$. Hence for each point $y$ in $W=$ $(h \circ \pi)^{-1}\left(z_{0}\right)$, the differential $d_{y}(h \circ \pi): T_{y} Y \rightarrow T_{z_{0}} \mathbb{S}^{p}$ is surjective and induces a linear isomorphism from the normal space of $W$ in $Y$ at $y$ onto the normal space of $z_{0}$ in $\mathbb{S}^{p}$. Consequently, there exists a unique smooth map $g: B(Y, W) \rightarrow B\left(\mathbb{S}^{p}, z_{0}\right)$ satisfying $h \circ \pi \circ \rho=\sigma \circ g$, where

$$
\rho=\pi(Y, W): B(Y, W) \rightarrow Y \quad \text { and } \quad \sigma=\pi\left(\mathbb{S}^{p}, z_{0}\right): B\left(\mathbb{S}^{p}, z_{0}\right) \rightarrow \mathbb{S}^{p}
$$

are the blowups (cf. [2, Lemma 2.5.9]). By construction, the diagram

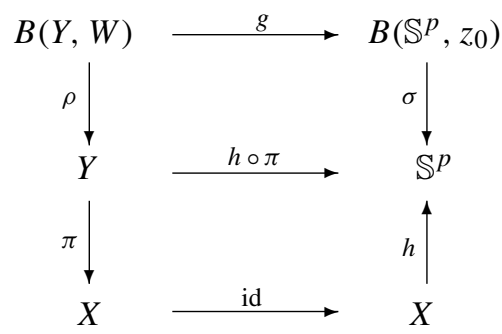


is commutative. The sets $D:=\rho^{-1}(W)$ and $E:=\sigma^{-1}\left(z_{0}\right)$ are nonsingular algebraic hypersurfaces in $B(Y, W)$ and $B\left(\mathbb{S}^{p}, z_{0}\right)$, respectively. Furthermore,

$$
D=g^{-1}(E) \quad \text { and } \quad g \text { is transverse to } E .
$$

Set $C:=\rho^{-1}\left(\pi^{-1}(S)\right)$. Since the restriction of $h \circ \pi: Y \rightarrow \mathbb{S}^{p}$ to $\pi^{-1}(S)$ is a regular map, and the restriction $\rho_{W}: B(Y, W) \backslash D \rightarrow Y \backslash W$ of $\rho$ and the restriction of $\sigma$ from $B\left(\mathbb{S}^{p}, z_{0}\right) \backslash E$ to $\mathbb{S}^{p} \backslash\left\{z_{0}\right\}$ are biregular isomorphisms, in view of (2), it follows that

$$
\text { the restriction }\left.g\right|_{C}: C \rightarrow B\left(\mathbb{S}^{p}, z_{0}\right) \text { is a regular map. }
$$

Moreover,

$$
C \cap D=\emptyset .
$$

Let $u$ be the cohomology class in $H^{1}(B(Y, W) ; \mathbb{Z} / 2)$ corresponding via the Poincaré duality to the homology class in $H_{*}(B(Y, W) ; \mathbb{Z} / 2)$ represented by $D$. By definition, $u$ belongs to $H_{\mathrm{alg}}^{1}(B(Y, W) ; \mathbb{Z} / 2)$. Similarly, let $v$ be the cohomology class in $H^{1}\left(B\left(\mathbb{S}^{p}, z_{0}\right) ; \mathbb{Z} / 2\right)$ Poincaré dual to the homology class in $H_{*}\left(B\left(\mathbb{S}^{p}, z_{0}\right) ; \mathbb{Z} / 2\right)$ represented by $E$. Recall that $B\left(\mathbb{S}^{p}, z_{0}\right)$ is biregularly isomorphic to $\mathbb{P}^{p}(\mathbb{R})$. In fact, there exists a biregular isomorphism

$$
\varphi: B\left(\mathbb{S}^{p}, z_{0}\right) \rightarrow \mathbb{P}^{p}(\mathbb{R}) \quad \text { with } \quad \varphi(E)=\mathbb{P}^{p-1}(\mathbb{R}),
$$

where $\mathbb{P}^{p-1}(\mathbb{R})$ is regarded as a subset of $\mathbb{P}^{p}(\mathbb{R})$. In particular, the group $H^{1}\left(B\left(\mathbb{S}^{p}, z_{0}\right) ; \mathbb{Z} / 2\right) \cong \mathbb{Z} / 2$ is generated by $v$. Condition (3) implies the equality

$$
u=g^{*}(v)
$$

(cf. [15, Proposition 2.15]), and hence

$$
g^{*}\left(H^{1}\left(B\left(\mathbb{S}^{p}, z_{0}\right) ; \mathbb{Z} / 2\right)\right) \subseteq H_{\mathrm{alg}}^{1}(B(Y, W) ; \mathbb{Z} / 2) .
$$

Let $i: D \hookrightarrow B(Y, W)$ and $j: E \hookrightarrow B\left(\mathbb{S}^{p}, z_{0}\right)$ be the inclusion maps. The restriction $\bar{g}: D \rightarrow E$ of $g$ satisfies $g \circ i=j \circ \bar{g}$, and hence

$$
i^{*}(u)=i^{*}\left(g^{*}(v)\right)=\bar{g}^{*}\left(j^{*}(v)\right) .
$$

In view of (6), the cohomology group $H^{1}(E ; \mathbb{Z} / 2)$ is generated by $j^{*}(v)$. Since the cohomology class $i^{*}(u)$ belongs to $H_{\text {alg }}^{1}(D ; \mathbb{Z} / 2)$, one gets

$$
\bar{g}^{*}\left(H^{1}(E ; \mathbb{Z} / 2)\right) \subseteq H_{\text {alg }}^{1}(D ; \mathbb{Z} / 2) .
$$

By (6), (8) and Lemma 2.2, there exists a regular map $r: D \rightarrow E$ arbitrarily close to $\bar{g}: D \rightarrow E$ in $\mathcal{C}(D, E)$.

In view of (5), there exists a smooth map $G: B(Y, W) \rightarrow B\left(\mathbb{S}^{p}, z_{0}\right)$ with

$$
\left.G\right|_{C}=\left.g\right|_{C} \text { and }\left.G\right|_{D}=j \circ r .
$$


Moreover, such a map $G$ can be chosen arbitrarily close to $g$, provided that $r$ is sufficiently close to $\bar{g}$. Note that

$$
G(D) \subseteq E .
$$

Setting $A:=C \cup D$, in view of (4), (5) and (9), one concludes that the restriction $\left.G\right|_{A}: A \rightarrow B\left(\mathbb{S}^{p}, z_{0}\right)$ is a regular map. Hence by (6), (7) and Lemma 2.2 , there exists a regular map $F: B(Y, W) \rightarrow B\left(\mathbb{S}^{p}, z_{0}\right)$ which is arbitrarily close to $G$ and satisfies $\left.F\right|_{A}=\left.G\right|_{A}$. In particular, according to (9),

$$
\left.F\right|_{C}=\left.g\right|_{C}
$$

Moreover, (10) implies the equality

$$
(\sigma \circ F)(D)=\left\{z_{0}\right\} .
$$

It follows from (12) that there exists a unique continuous map $\bar{F}: Y \rightarrow \mathbb{S}^{p}$ satisfying $\bar{F} \circ \rho=\sigma \circ F$. Explicitly,

$$
\bar{F}(y)= \begin{cases}\sigma\left(F\left(\rho_{W}^{-1}(y)\right)\right) & \text { for } y \text { in } Y \backslash W, \\ z_{0} & \text { for } y \text { in } W .\end{cases}
$$

According to $(1),(h \circ \pi)(W)=\left\{z_{0}\right\}$. Since $\sigma \circ F$ is close to $\sigma \circ g=h \circ \pi \circ \rho$, it follows that $\bar{F}$ is close to $h \circ \pi$. Observe that

$$
\bar{F}=h \circ \pi \quad \text { on } \pi^{-1}(S) .
$$

Indeed, in view of $(1), \pi^{-1}(S) \subseteq Y \backslash W$. If $y$ is a point in $\pi^{-1}(S)$, then the point $\rho_{W}^{-1}(y)$ is in $\rho^{-1}\left(\pi^{-1}(S)\right)=C$, and hence (11) and (13) imply

$$
\bar{F}(y)=(\sigma \circ g)\left(\rho_{W}^{-1}(y)\right)=(h \circ \pi \circ \rho)\left(\rho_{W}^{-1}(y)\right)=(h \circ \pi)(y),
$$

which proves (14).

Condition (14) implies that the map $f: X \rightarrow \mathbb{S}^{p}$ defined by

$$
f(x)= \begin{cases}\bar{F}\left(\pi_{S}^{-1}(x)\right) & \text { for } x \text { in } X \backslash S, \\ h(x) & \text { for } x \text { in } S,\end{cases}
$$

is continuous. The map $f$ is close to $h$ since $\bar{F}$ is close to $h \circ \pi$. It can be assumed that $f$ is in $\mathcal{U}$. By (1), if $x$ is a point in $\operatorname{Reg}(V)=h^{-1}\left(z_{0}\right)$, then the point $\pi_{S}^{-1}(x)$ is in $W$, and hence (15) and (13) imply

$$
f(x)=\bar{F}\left(\pi_{S}^{-1}(x)\right)=z_{0}=h(x) .
$$

Since $V=S \cup \operatorname{Reg}(V)$, one gets $f(V)=h(V)$. Finally, $\pi^{-1}(X \backslash V) \subseteq Y \backslash W$, and hence (15) and (13) imply that

$$
\left.f\right|_{X \backslash V}=\bar{F} \circ\left(\left.\pi_{S}^{-1}\right|_{X \backslash V}\right)=\left(\sigma \circ F \circ \rho_{W}^{-1}\right) \circ\left(\left.\pi_{S}^{-1}\right|_{X \backslash V}\right),
$$

where $\bar{F}, \varrho_{W}^{-1}, F$ and $\sigma$ are considered suitably restricted. It follows that $\left.f\right|_{X \backslash V}$ is a regular map. Consequently, $f$ is a nice continuous rational map in $\mathcal{U}$, with $P(f) \subseteq V$ and $f(V)=h(V) \neq \mathbb{S}^{p}$. 
Remark 2.6. With notation as in Theorem 2.5 , one can estimate the size of $P(f)$ and $f(P(f))$. Namely,

$$
P(f) \subseteq h^{-1}\left(z_{0}\right) \cup S \quad \text { and } \quad f(P(f)) \subseteq\left\{z_{0}\right\} \cup h(S) .
$$

Furthermore, $h(S)=f(S)$ is a semi-algebraic set (cf. [5, Proposition 2.2.7]) and

$$
\operatorname{dim} h(S) \leq \operatorname{dim} S<\operatorname{dim} h^{-1}\left(z_{0}\right)=\operatorname{dim} X-p
$$

(cf. [5, Theorem 2.8.8] for the first inequality). In particular, if $\operatorname{dim} X=p$, then the set $h^{-1}\left(z_{0}\right)$ is finite, and hence it can be assumed that $V=h^{-1}\left(z_{0}\right)$ and $S=\emptyset$.

Proof of Theorem 1.2. In view of Proposition 1.1, condition (b) implies (c). By Lemma 2.4, (c) implies (d), and (e) implies (f). Clearly, (a) implies (b), (d) implies (c), (f) implies (e), and (d) implies (f).

Suppose that (d) is satisfied. For the proof of (a), one may assume without loss of generality that the map $h$ is smooth and adapted to a point $z$ in $\mathbb{S}^{p}$ with $h^{-1}(z)=\operatorname{Reg}(V)$ for some algebraic subset $V$ of $X$. The set $S:=V \backslash \operatorname{Reg}(V)$ is an algebraic subset of $X$ with $h(S) \subseteq \mathbb{S}^{p} \backslash\{z\}$. According to Lemma 2.3, the restriction map $\left.h\right|_{S}: S \rightarrow \mathbb{S}^{p}$ can be approximated in $\mathcal{C}\left(S, \mathbb{S}^{p}\right)$ by regular maps. Such regular maps are smooth, and hence in view of Lemma 2.4, the restriction map $\left.h\right|_{S}: S \rightarrow \mathbb{S}^{p}$ may be assumed to be regular. Applying Theorem 2.5, one concludes that condition (a) holds.

It remains to prove that (f) implies (d). Suppose that (f) is satisfied. For the proof of (d), one may assume that the map $h$ is weakly adapted to a point $z_{0}$ in $\mathbb{S}^{p}$. Hence the smooth submanifold $h^{-1}\left(z_{0}\right)$ of $X$ admits a weak algebraic approximation in $X$. It follows that $h^{-1}\left(z_{0}\right)$ is isotopic in $X$, via an arbitrarily small smooth isotopy, to $\operatorname{Reg}(W)$ for some algebraic subset $W$ of $X$. Such an isotopy can be extended to a smooth diffeotopy of $X$, close to the identity map of $X$ in the space $\mathcal{C}^{\infty}(X, X)$ (cf. [22, pp. 179, 180]). In particular, there exists a smooth diffeomorphism $\varphi: X \rightarrow X$ such that $\varphi\left(h^{-1}\left(z_{0}\right)\right)=\operatorname{Reg}(W)$ and the composite map $h \circ \varphi^{-1}$ is close to $h$ in the space $\mathcal{C}\left(X, \mathbb{S}^{p}\right)$. By construction, the map $h \circ \varphi^{-1}$ is smooth and transverse to $z_{0}$, and $\left(h \circ \varphi^{-1}\right)^{-1}\left(z_{0}\right)=\operatorname{Reg}(V)$. Consequently, condition (d) holds, as required.

In view of Theorem 1.2 and Corollary 1.4, it is desirable to have a criterion for a smooth submanifold to admit an almost algebraic approximation. It suffices to consider submanifolds embedded with trivial normal bundle. If $P$ is a smooth manifold with boundary, its boundary will be denoted by $\partial P$.

Proposition 2.7. Let $X$ be a compact nonsingular real algebraic variety and let $M$ be a compact smooth submanifold of $X$. Assume that there exists an algebraic subset $A$ of $X$ such that $M \cap \operatorname{Reg}(A)=\emptyset$ and

$$
M \cup \operatorname{Reg}(A)=\partial P,
$$

where $P$ is a compact smooth manifold with boundary, embedded in $X$ with trivial normal bundle and satisfying $P \cap A=\operatorname{Reg}(A)$. Then $M$ admits a weak algebraic approximation in $X$. 
Proof. There exists a multiblowup $\pi: Y \rightarrow X$ of $X$ over $S:=A \backslash \operatorname{Reg}(A)$ such that the subset $B=\pi^{-1}(\operatorname{Reg}(A))$ of $Y$ is Zariski closed and nonsingular (cf. the beginning of the proof of Theorem 2.5). Set $N:=\pi^{-1}(M)$. Since the restriction $\pi_{S}: Y \backslash \pi^{-1}(S) \rightarrow X \backslash S$ of $\pi$ is a biregular isomorphism and $P$ is disjoint from $S$, the set $Q:=\pi^{-1}(P)$ is a compact smooth manifold with boundary $\partial Q=N \cup B$, embedded in $Y$ with trivial normal bundle. It follows that there exists a smooth embedding $\varepsilon: N \rightarrow Y$, arbitrarily close to the inclusion map $N \hookrightarrow Y$ in the space $\mathcal{C}^{\infty}(N, Y)$, with $\varepsilon(N) \subseteq Y \backslash \pi^{-1}(S)$ and $\varepsilon(N)=W$ for some nonsingular algebraic subset $W$ of $Y$ (cf. [25, Lemma 2.3]). The map $e: M \rightarrow X$ defined by $e(x)=\pi\left(\varepsilon\left(\pi_{S}^{-1}(x)\right)\right)$ for all $x$ in $M$ is a smooth embedding, close to the inclusion map $M \hookrightarrow X$ in the space $\mathcal{C}^{\infty}(M, X)$. Furthermore, $e(M)=\pi(W)$. By construction, $\pi(W)$ is a nonsingular Zariski closed subset of $X \backslash S$. Consequently, $\pi(W)=\operatorname{Reg}(V)$, where $V$ is the Zariski closure of $\pi(W)$ in $X$. The proof is complete.

The next result is of a different nature, but closely related to theorems of Section 1. As before, $s_{p}$ will stand for the unique generator of the cohomology group $H^{p}\left(\mathbb{S}^{p} ; \mathbb{Z} / 2\right)$, $p \geq 1$. For any compact smooth manifold $M$ of dimension $n$, let $[M]$ denote its fundamental class in $H_{n}(M ; \mathbb{Z} / 2)$. If $K$ is a compact smooth $p$-dimensional submanifold of $M$, let $[K]_{M}$ denote the homology class in $H_{p}(M ; \mathbb{Z} / 2)$ represented by $K$.

Theorem 2.8. Let $M$ be a compact smooth manifold and let $p$ be an integer satisfying $0<p<\operatorname{dim} M$. Assume that $M$ is stably parallelizable and the homology group $H_{p}(M ; \mathbb{Z} / 2)$ is generated by homology classes represented by compact smooth p-dimensional submanifolds of $M$ with trivial normal bundle. For any continuous map $h: M \rightarrow \mathbb{S}^{p}$ with $h^{*}\left(s_{p}\right) \neq 0$ in $H^{p}(M ; \mathbb{Z} / 2)$, there exist a nonsingular irreducible real algebraic variety $X$ and a smooth diffeomorphism $\varphi: X \rightarrow M$ such that the map $h \circ \varphi: X \rightarrow \mathbb{S}^{p}$ is not homotopic to a continuous rational map.

Proof. Set $v:=h^{*}\left(s_{p}\right)$. By assumption and the universal coefficient theorem,

$$
\left\langle v,[K]_{M}\right\rangle \neq 0
$$

for some compact smooth $p$-dimensional submanifold $K$ of $M$ with trivial normal bundle. Since $M$ is stably parallelizable, the Stiefel-Whitney classes of $K$ are all zero (cf. [28, p. 41]). Hence by Thom's theorem [29], $K$ is the boundary of a compact smooth manifold with boundary. These properties of $K$ make it possible to apply [13, Proposition 2.5, Theorem 2.6]. Therefore there exist a nonsingular irreducible real algebraic variety $X$ and a smooth diffeomorphism $\varphi: X \rightarrow M$ such that the homology class $\left[\varphi^{-1}(K)\right]_{X}$ in $H_{p}(X ; \mathbb{Z} / 2)$ satisfies

$$
\left\langle u,\left[\varphi^{-1}(K)\right]_{X}\right\rangle=0
$$

for every cohomology class $u$ in $H_{\mathrm{alg}}^{p}(X ; \mathbb{Z} / 2)$. In view of (i), (ii) and the equalities

$$
\begin{aligned}
\left\langle(h \circ \varphi)^{*}\left(s_{p}\right),\left[\varphi^{-1}(K)\right]_{X}\right\rangle & =\left\langle\varphi^{*}\left(h^{*}\left(s_{p}\right)\right),\left[\varphi^{-1}(K)\right]_{X}\right\rangle=\left\langle h^{*}\left(s_{p}\right), \varphi_{*}\left(\left[\varphi^{-1}(K)\right]_{X}\right)\right\rangle \\
& =\left\langle v,[K]_{M}\right\rangle,
\end{aligned}
$$


the cohomology class $(h \circ \varphi)^{*}\left(s_{p}\right)$ is not in $H_{\text {alg }}^{p}(X ; \mathbb{Z} / 2)$. The functoriality of $H_{\text {alg }}^{p}(-; \mathbb{Z} / 2)$ implies that the map $h \circ \varphi$ cannot be homotopic to a continuous rational map (cf. Section 1).

Corollary 2.9. Let $M$ be a compact smooth manifold of dimension $p+1$, where $p \geq 1$. Assume that $M$ is stably parallelizable and the cohomology group $H^{2}(M ; \mathbb{Z})$ has no 2torsion. For any continuous map $h: M \rightarrow \mathbb{S}^{p}$ with $h^{*}\left(s_{p}\right) \neq 0$ in $H^{p}(M ; \mathbb{Z} / 2)$, there exist a nonsingular irreducible real algebraic variety $X$ and a smooth diffeomorphism $\varphi: X \rightarrow M$ such that the map $h \circ \varphi: X \rightarrow \mathbb{S}^{p}$ is not homotopic to a continuous rational map.

Proof. By Theorem 2.8, it suffices to prove that each homology class $\alpha$ in $H_{p}(M ; \mathbb{Z} / 2)$ can be represented by a compact smooth $p$-dimensional submanifold of $M$ with trivial normal bundle. Let $w$ be the cohomology class in $H^{1}(M ; \mathbb{Z} / 2)$ Poincaré dual to $\alpha$. Since the group $H^{2}(M ; \mathbb{Z})$ has no 2-torsion, by the universal coefficient theorem, the reduction modulo 2 homomorphism $r: H^{1}(M ; \mathbb{Z}) \rightarrow H^{1}(M ; \mathbb{Z} / 2)$ is surjective. Let $\bar{w}$ be a cohomology class in $H^{1}(M ; \mathbb{Z})$ with $r(\bar{w})=w$. There exists a continuous map $g: M \rightarrow S^{1}$ with $\bar{w}=g^{*}\left(\bar{s}_{1}\right)$, where $\bar{s}_{1}$ is a generator of the group $H^{1}\left(\mathbb{S}^{1} ; \mathbb{Z}\right) \cong \mathbb{Z}$ (cf. [16, p. 492]). In the case under consideration, the map $g$ can be assumed to be smooth. By Sard's theorem, $g$ is transverse to a point $z_{0}$ in $\mathbb{S}^{1}$. The normal bundle of the smooth submanifold $K:=g^{-1}\left(z_{0}\right)$ of $M$ is trivial. Furthermore, the homology class $[K]_{M}$ in $H_{p}(M ; \mathbb{Z} / 2)$ is Poincaré dual to $w$ (cf. [15, Proposition 2.15]), and hence $\alpha=[K]_{M}$.

Acknowledgements. The author was partially supported by NCN grant 2011/01/B/ST1/01289.

\section{References}

[1] Akbulut, S., King, H.: On approximating submanifolds by algebraic sets and a solution to the Nash conjecture. Invent. Math. 107, 87-97 (1992) Zbl 0781.14038 MR 1135465

[2] Akbulut, S., King, H.: Topology of Real Algebraic Sets. Math. Sci. Res. Inst. Publ. 25, Springer (1992) Zbl 0808.14045 MR 1225577

[3] Benedetti, R., Tognoli, A.: Remarks and counterexamples in the theory of real algebraic vector bundles and cycles. In: Géométrie algébrique réelle et formes quadratiques, Lecture Notes in Math. 959, Springer, 198-211 (1982) Zbl 0498.14015 MR 0683134

[4] Bochnak, J., Buchner, M., Kucharz, W.: Vector bundles over real algebraic varieties. K-Theory 3, 271-298 (1989) Zbl 0761.14020 MR 1040403

[5] Bochnak, J., Coste, M., Roy, M.-F.: Real Algebraic Geometry. Ergeb. Math. Grenzgeb. 36, Springer (1998) Zbl 0912.14023 MR 1659509

[6] Bochnak, J., Kucharz, W.: Algebraic approximation of mappings into spheres. Michigan Math. J. 34, 119-125 (1987) Zbl 0631.14019 MR 0873026

[7] Bochnak, J., Kucharz, W.: Realization of homotopy classes by algebraic mappings. J. Reine Angew. Math. 377, 159-169 (1987) Zbl 0619.14014 MR 0887406

[8] Bochnak, J., Kucharz, W.: On real algebraic morphisms into even-dimensional spheres. Ann. of Math. (2) 128, 415-433 (1988) Zbl 0674.14013 MR 0960952

[9] Bochnak, J., Kucharz, W.: Algebraic models of smooth manifolds. Invent. Math. 97, 585-611 (1989) Zbl 0687.14023 MR 1005007 
[10] Bochnak, J., Kucharz, W.: The homotopy groups of some spaces of real algebraic morphisms. Bull. London Math. Soc. 25, 385-392 (1993) Zbl 0799.57015 MR 1222733

[11] Bochnak, J., Kucharz, W.: Elliptic curves and real algebraic morphisms. J. Algebraic Geom. 2, 635-666 (1993) Zbl 0807.14046 MR 1227471

[12] Bochnak, J., Kucharz, W.: On homology classes represented by real algebraic varieties. In: Singularities Symposium- Łojasiewicz 70, Banach Center Publ. 44, Inst. Math., Polish Acad. Sci., Warszawa, 21-35 (1998) Zbl 0915.14033 MR 1677394

[13] Bochnak, J., Kucharz, W.: Real algebraic morphisms represent few homotopy classes. Math. Ann. 337, 909-921 (2007) Zbl 1154.14042 MR 2285743

[14] Bochnak, J., Kucharz, W., Silhol, R.: Morphisms, line bundles and moduli spaces in real algebraic geometry. Inst. Hautes Études Sci. Publ. Math. 86, 5-65 (1997) Zbl 0938.14033 MR 1608561

[15] Borel, A., Haefliger, A.: La classe d'homologie fondamentale d'un espace analytique. Bull. Soc. Math. France 89, 461-513 (1961) Zbl 0102.38502 MR 0149503

[16] Bredon, G. E.: Topology and Geometry. Springer (1993) Zbl 0791.55001 MR 1224675

[17] Fichou, G., Huisman, J., Mangolte, F., Monnier, J.-Ph.: Fonctions régulues. J. Reine Angew. Math., to appear; arXiv:1112.3800

[18] Ghiloni, R.: Rigidity and moduli space in real algebraic geometry. Math. Ann. 335, 751-766 (2006) Zbl 1098.14045 MR 2232015

[19] Ghiloni, R.: On the space of morphisms into generic real algebraic varieities. Ann. Scuola Norm. Sup. Pisa Cl. Sci. (5) 5, 419-438 (2006) Zbl 1170.14309 MR 2274786

[20] Ghiloni, R.: Second order homology obstructions on real algebraic manifolds. Topology Appl. 154, 3090-3094 (2007) Zbl 1122.14038 MR 2355513

[21] Hironaka, H.: Resolutions of singularities of an algebraic variety over a field of characteristic zero. Ann. of Math. (2) 79, 109-326 (1964) Zbl 0122.38603 MR 0199184

[22] Hirsch, M. W.: Differential Topology. Springer (1997)

[23] Kollár, J.: Lectures on Resolution of Singularities. Ann. of Math. Stud. 166, Princeton Univ. Press, Princeton, NJ (2007) Zbl 1113.14013 MR 2289519

[24] Kollár, J., Nowak, K.: Continuous rational functions on real and $p$-adic varieties. Math Z., online (2014); doi:10.1007/s00209-014-1358-7

[25] Kucharz, W.: On homology of real algebraic sets. Invent. Math. 82, 19-25 (1985) Zbl 0547.14018 MR 0808106

[26] Kucharz, W.: Rational maps in real algebraic geometry. Adv. Geom. 9, 517-539 (2009) Zbl 1173.14341 MR 2574137

[27] Kucharz, W.: Complex cycles on algebraic models of smooth manifolds. Math. Ann. 346, 829-856 (2010) Zbl 1222.14126 MR 2587094

[28] Milnor, J. W., Stasheff, J. D.: Characteristic Classes. Ann. of Math. Stud. 76, Princeton Univ. Press, Princeton, NJ (1974) Zbl 0298.57008 MR 0440554

[29] Thom, R.: Quelques propriétés globales des variétés différentiables. Comment. Math. Helv. 28, 17-86(1954) Zbl 0057.15502 MR 0061823 